\title{
National Cancer Institute, AlIMS, Delhi: A Pathbreaking Project to Bring in a New Era in Cancer Care in India
}

With the control of communicable diseases, noncommunicable diseases (NCDs) including cancers are fast becoming major causes of morbidity and mortality in India. Cancers have profound social, psychological and financial implications on the patients and their families. In India, it often leads to family impoverishment, physical and mental distress. In our country, the data on cancer in India is collected by Population Based Cancer Registries (PBCRs) of the National Cancer Registry Programme (NCRP) of Indian Council of Medical Research (ICMR). Realizing the success of the NCRP, it was extended to cover other common NCDs such as diabetes, CVD and stroke and was converted to National Centre for Disease Informatics and Research (NCDIR) under the ICMR in March 2011, because most NCDs have similar etiological factors. The broad and overall objective of the NCDIR is to sustain and develop a national research database of four common NCDs through recent advances in electronics and information technology with a national collaborative network. According to the NCDIR data, annual incidence of cancer which was 14.5 lakhs in 2016 is projected to be 17.5 lakhs by 2020. Breast

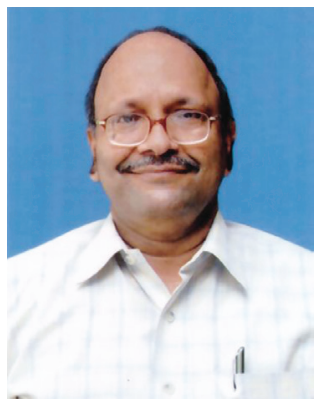
cancer incidence is expected to increase from 1.5 lakhs to 1.9 lakhs, whereas lung cancer incidence will rise from 1.14 lakhs to 1.4 lakhs by 2020 . The cancer death per year is projected to increase from 7.36 lakhs to 8.8 lakhs in 2020 . However, there is a wide demographic variation in the incidence of cancer across the country. Aizawl district in Mizoram has the highest age-adjusted rate (AAR) of 270.7 in males while the rural registry of Barshi in Maharashtra has the lowest AAR of 40.9 per 100,000 population. This suggests the impact of varied environmental, genetic and socio-cultural factors like tobacco use, infections, and other modifiable risk factors. AAR in India are, however, lower compared with the Western data. Significant progress is happening in the areas of avoidable cancer. Cervical cancer incidence is coming down in India. Besides this, the recent WHO report has shown that the tobacco smoking reduction target of 2025 has already been achieved by India. Significant progress and achievements have also happened in the treatment of cancer in different parts of the world. The cure rates of some of the cancers like prostate have reached nearly $100 \%$ in early stages. Some cancers like breast, melanoma and thyroid have reached a 5 -year survival rate of $90 \%$ or more. The above achievements have been possible due to better understanding of the biology of cancer and results of translational research in the various aspects of oncology carried out all over the world. The three major publications from NCDIR on patterns of care and survival studies (POCSS), one each on breast, head and neck and cervical cancer document the magnitude problem of cancer in our country and the results of cancer treatment in our country.

The government and private sectors both have been continuously working towards infrastructure development and capacity building in cancer care in India. The National Cancer Control Programme (NCCP) is a centrally sponsored scheme by the Ministry of Health and Family Welfare, Government of India (Gol), which was initiated in 1975. Initial priority of this programme was to equip the premier cancer hospitals/institutions in India to make them capable of treating cancer. Later, more emphasis was placed on primary prevention and early detection of cancer with modifications in the year 1984. The District Cancer Control Programme (DCCP) was introduced during 1990-1991 and later modified in 2000-2001. NCCP was evaluated in 2004, and was further revised with effect from 2005.

In view of preventable common risk factors of cancer and other NCDs, the Gol also started the National Programme for Prevention and Control of Cancers, Diabetes, Cardiovascular Diseases and Stroke (NPCDCS) after integrating the NCCP with other NCDs. Under the NPCDCS, there is an allocation of 120 crores to each for the establishment of 20 State Cancer Institutes (SCl). A sum of $45 \mathrm{crores}$ each has also been earmarked for 50 Tertiary Care Cancer Centers (TCCC). A total of 35 institutions across the country have been benefited under this program so far. Under the earlier NCCP, schemes like regional cancer centre, oncology wing in medical colleges and DCCP, augmentation of infrastructure of several institutions have been implemented. Significant achievements have been made through these planned activities and the geographical gap in the treatment facilities in the country has been reduced.

The delivery of affordable and equitable cancer care is one of our greatest public health challenges. Out-of-pocket payments are a big burden to the patients and their families. The Gol has recently launched a large scheme for the poor people of the country called National Health Protection Mission, also referred to as Ayushman Bharat or the Pradhan Mantri Jan Arogya Yojana. This scheme is the largest health insurance project so far. It has two main pillars: strengthening of universal comprehensive primary health care and a health insurance scheme to cover 500 million people in need to reduce catastrophic out-of-pocket health spending.

We presently need to invest in suitable surveillance systems to monitor the changing trends in NCDs. Cancer registration program, which is well regarded, needs further strengthening and extension to the states not covered particularly the large ones. This includes recording of cause of death reporting system. So, presently we have an increasing incidence of cancer with negative socioeconomic impact, poor coordination among various cancer centers in treatment and an even greater need to conduct innovative and well-coordinated research to improve cancer outcomes in our country.

The National Cancer Institute ( $\mathrm{NCl}$ ), in the second campus of AIIMS, in Jhajjar, Haryana, has been envisaged as an institute of national importance. The main focus will be on translational cancer research particularly for India-centric ones and formulation of modern therapy for cancer patients in India besides providing state-of-the-art cancer care at very low costs. The state-of-the-art center was dedicated to the nation on 12th February, 2019, by the honorable prime minister of India. When fully commissioned, it will have 710 beds and 2705 employees. The research wing will contain 15 departments and 200 beds dedicated for translational research. The $\mathrm{NCl}$ is spread over an area of 67 acres in a campus of 300 acres with all the departments to conduct research. Various research projects as envisaged in the INDO-US Memorandum of Understandings have already been initiated in very close coordination with the main campus at AlIMS, New Delhi. The major objectives of this center is also to provide affordable quality tertiary cancer care to cancer patients, to act as the 
principal agency of the country for coordinating and conducting research in oncology, to undertake clinical trials of newer drugs as well as vaccines to treat and improve the existing outcomes of therapy on survival and quality of life of the cancer patients, to set standards for cancer treatment and to evolve, update and disseminate evidence-based guidelines to promote uniform patient care across the country, to develop and support training programs and generate trained human resources in all areas of cancer who can participate in basic and epidemiological research and cancer treatment programs, to support and coordinate quality cancer research in regional cancer centers, other governmental and nongovernmental agencies. It will also strive to improve and harmonize the quality of research in cancer across the country and to translate the knowledge gained from research to evolve national cancer control policies and programs to improve the quality of cancer care services and quality of life of cancer patients. Emphasis will be placed towards creation of an independent datamonitoring committee to monitor and intervene if necessary in various trials in the country. NCl, in coordination with AllMS, has already started conducting bench to bedside research. We also wish to start a new degree called MD-PhD in due course of time, which will be a comprehensive 6 years integrated training program. At the end of this course, a new group of clinical oncologists will be available in the country every year. They will not only understand the disease better, but will also contribute to eradicate cancer by conducting both fundamental and clinical research in the various areas like prevention, early diagnosis, management and rehabilitation. Like the success of the Indian Space Research Organization, we are very confident that the NCI project will be successful in eradicating cancer in the long run.

Prof G K Rath

Head, National Cancer Institute, AlIMS Ansari Nagar, New Delhi, India 